\title{
Magnetization Reversal Phenomena and Domain Wall Behaviors in Nanostructured Magnetic Systems
}

\author{
T. SHINJo \\ Institute for Chemical Research, Kyoto University \\ Uji, Kyoto-fu 611-0011, Japan
}

\begin{abstract}
Several recent experiments on micro- (or nano-) structured samples of ferromagnetic materials are introduced. Magnetization reversal phenomena are investigated on submicron wire samples of trilayer structure using the giant magnetoresistance effect. Domain wall movements are sensitively monitored by resistivity measurements and the velocity of propagation is determined. The contribution of domain wall to the resistivity is argued from the results on artificially designed samples of a spring-magnet system. In circular dots of permalloy, the existence of vortex magnetization is confirmed and the reversal of the vortex core magnetization is studied from magnetic force microscopy measurements.
\end{abstract}

PACS numbers: 75.50.Tt, 75.75.+a

\section{Introduction}

It is expected that magnetic properties are controlled by structural engineering on nanoscale and novel magnetic phenomena may be exhibited in nanostructured systems. Micro- (or nano-) fabrication techniques have been utilized routinely in the field of semiconductor physics to prepare mesoscopic systems. For metallic and magnetic systems also, the use of nanofabrication techniques has become popular recently. The author's group is using an electron-beam lithography equipment to prepare submicron wires and dots of ferromagnetic materials. In the present article, several experimental studies relating to magnetization reversal phenomena and domain wall behaviors in nanomagnetic systems are described.

Magnetization reversal phenomena in nanoscale magnetic systems have renewed interests from basic and also technical viewpoints. For instance, the possibility of macroscopic quantum tunneling (MQT) process was predicted theoretically 
if the size of the magnetic system is extremely small [1]. Pioneering studies on MQT in mesoscopic systems have been reported by several groups [2]. In an ultrathin wire of ferromagnetic material, the direction of magnetization is limited so that it is parallel to the wire axis because of the shape anisotropy. If there is only one domain wall, the magnetization reversal is regarded as propagation of the domain wall. In case that the cross-section of the wire is very small, the domain wall size also should be a mesoscopic scale and the domain wall is expected to behave as a quasiparticle. It is of great interest to observe the properties of domain wall in a very narrow wire. The author's group has prepared single wire systems consisting of two magnetic layers with different coercivities and succeeded in observing domain wall movements utilizing the giant magnetoresistance (GMR) effect, as described in the next section.

In the region of domain wall, spin directions of each atom vary gradually and a non-collinear spin structure is formed. The disordered spin structure may influence the conductivity. However, the contribution of a domain wall to the resistivity is normally very small compared with anisotropic magnetoresistance (AMR) and still is an open question. Very recently it has been suggested that the magnetoresistance in point-contact systems may be extremely large even at room temperature $[3,4]$. In the third section, experiments to estimate the resistivity of domain wall in a sample of spring-magnet system are introduced.

In the last place, recent results in circular permalloy dots are presented. By using magnetic force microscopy (MFM), it is confirmed that the magnetization in a dot is forming a vortex structure and a turned-off magnetization spot exists at the core of the vortex. Behaviors of turned-off magnetization spots under external fields are studied by comparing MFM results and magnetization measurements by SQUID magnetometer.

\section{Magnetization reversal in submicron wire}

Magnetization in a single wire sample with submicron width is too small to measure by normal experimental techniques and therefore it is not easy to study magnetization reversal phenomena in nanoscale systems. A possibility to detect magnetization reversal in a nanoscale wire is resistivity mesurements utilizing the GMR effect [5]. Samples for resistivity measurements having a pattern shown in Fig. 1a were prepared by electron-beam lithography with lift-off method. The content is the following trilayer structure, $\mathrm{NiFe}(2 \mathrm{~nm}) / \mathrm{Cu}(2 \mathrm{~nm}) / \mathrm{Co}(2 \mathrm{~nm})$, and the wire width is $150 \mathrm{~nm}$. The distance between the leads for resistivity measurements is $20 \mu \mathrm{m}$. The resistivity as a function of the applied field at $300 \mathrm{~K}$ is shown in Fig. 1b. The pad, with a large area, $0.5 \times 0.5 \mu \mathrm{m}$, is attached at one end of the wire to specify the nucleation site of magnetization reversal. Prior to the measurement, a magnetic field of 100 Oe was applied in order to align all magnetization parallel. Then the resistivity was measured with sweeping the external field. Since the 
(a)
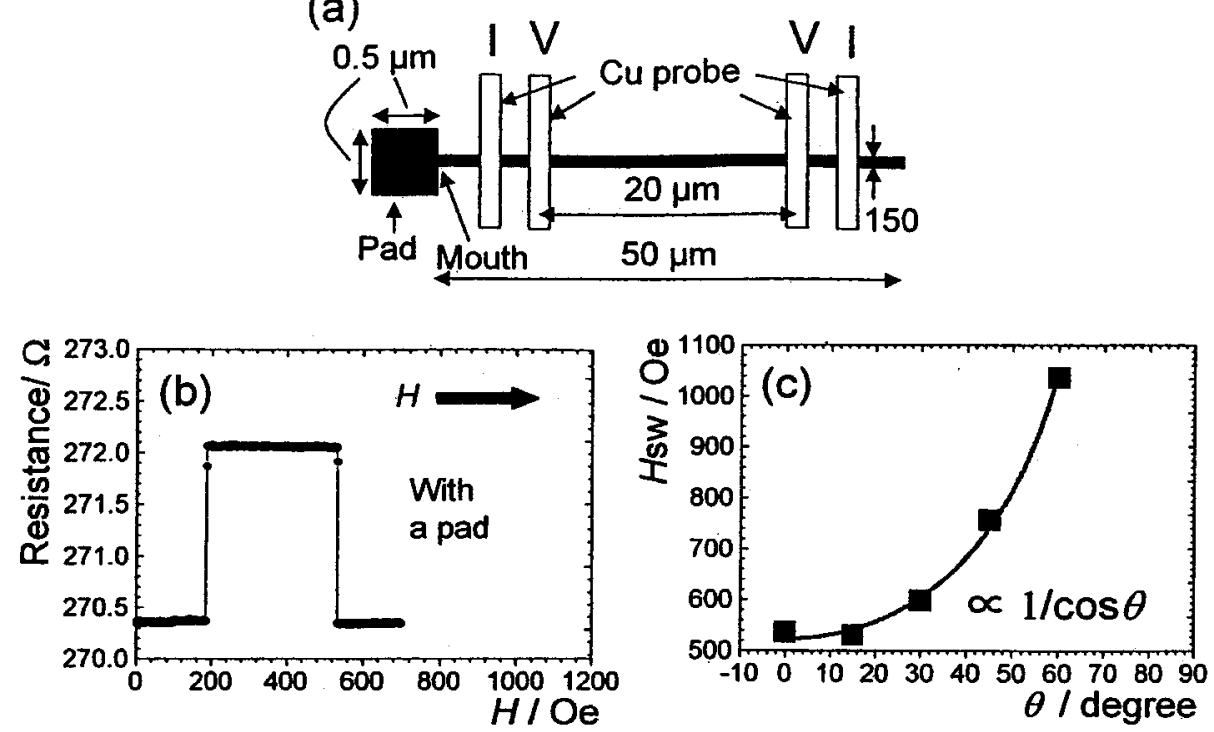

Fig. 1. (a) Schematic illustration of wire sample with the composition of $\operatorname{NiFe}(2 \mathrm{~nm}) / \mathrm{Cu}(2 \mathrm{~nm}) / \mathrm{Co}(4 \mathrm{~nm})$ with a pad at one end. (b) Resistance as a function of external magnetic field. (c) Angular dependence of the switching field for the Co layer at $300 \mathrm{~K}$. The angle $\theta$ denotes the external field direction relative to the wire axis.

coercive field for the NiFe layer is smaller than that for the Co layer, the reversal of NiFe magnetization occurs first and eventually two magnetizations are aligned antiparallel. Accordingly, the resistivity shows a sudden increase due to the GMR effect. If the field reaches to the coercive field of the Co layer, magnetizations of the two layers again become parallel and the resistivity has come back to the ground value.

It is to be noted that abrupt changes of resistivity are observed only at the moments of magnetization reversal. This result suggests that the magnetization reversal takes place with a single domain wall propagation and the velocity is very fast. A preliminary estimation of the velocity of the domain wall propagation was carried out and the velocity was actually derived to be in the order of $100 \mathrm{~m} / \mathrm{s}$ [6]. The reversal process is considered as follows. The nucleation starts at the pad area but the domain wall is trapped at the connecting part between the pad and wire ("mouth" in Fig. 1a). The switching field of the magnetic layer corresponds to the critical value for the trapped domain wall at the mouth. If external field attains the critical value, the domain wall starts to propagate. This model is verified by the measurement with changing the direction of external fields relative to the wire axis (Fig. 1c). With increase in the angle $\theta$, the critical field $H_{\mathrm{sw}}$ increases but $H_{\mathrm{sw}} \cos \theta$ is found to be constant. The domain wall propagation means that the magnetic field, in the wire direction, reaches a certain value, which is a potential barrier 
for a domain wall trapped at the mouth. The measurements on the temperature dependence of the switching field are in progress [7].

\section{Resistivity of domain wall}

In the preceding section, studies on domain walls utilizing GMR effect were described. In these resistivity measurements, the electric currents are mainly flowing in the spacer (non-magnetic) layers and the contribution of domain wall itself to the resistivity is negligible. In a domain wall region of ferromagnet, spins are changing the directions gradually and a kind of non-collinear spin arrangement is realized. An interesting issue is what the contribution of domain wall to the resistance is. There have been several experiments to study the domain wall resistance in ferromagnetic wire samples [8]. However, because the absolute resistance of domain wall is very small and anisotropic magnetoresistance (AMR) is dominating, a conclusive result has not yet been obtained. Even whether the existence of domain walls enhances or reduces the conductivity is still an open question. The author's group has prepared model systems to study the contribution of domain wall to the conductivity by utilizing an exchange spring system, which consists of soft magnetic (NiFe) and hard magnetic (CoSm) layers [9]. In an exchange spring system, the magnetic moments in the soft layer are pinned by the hard magnetic layer at the interface. When an inverse magnetic field is applied to the saturated state along the easy direction, the magnetic moments in the soft layer rotate and the directions are distributed as illustrated in Fig. 2a. The magnetic structure in the soft layer with gradually rotating moments is similar to the spin structure in a Bloch wall and therefore an exchange spring bilayer is regarded as a model system to investigate the resistance of a domain wall. The magnetoresistance of a NiFe/CoSm bilayer, measured in the current-in-plane geometry, is shown in Fig. 2b. Since the resistivity of CoSm is much larger than $\mathrm{NiFe}$, the electric current mostly flows in the NiFe layer. However, NiFe layer shows a significantly large AMR effect. In order to remove the influence from the AMR effect, the magnetoresistance has been measured with two current directions relative to the field direction, and the obtained results (Fig. 2b) are superposed to cancel the AMR effect. Then, as a result, the resistivity as shown in Fig. 2c is obtained, which is supposed to be the resistance due to the rotating spin structure similar to the Bloch wall. From this result, it is concluded that the contribution of non-uniform spin structure at a domain wall to the resistivity is positive but fairly small, less than $0.1 \%$.

Another approach to control the domain wall formation in a wire has been carried out using a similar system [10]. As shown in Fig. 3a, a wire of NiFe with attaching CoSm pads was prepared. The wire is $20 \mathrm{~nm}$ thick, $1 \mu \mathrm{m}$ width and $300 \mu \mathrm{m}$ length. CoSm pads are $40 \mathrm{~nm}$ thick and $30 \times 30 \mu \mathrm{m}$ area. After saturation, by applying a moderate field inversely, the major parts of NiFe layer change the magnetization direction while the covered parts by CoSm pads maintain the initial 


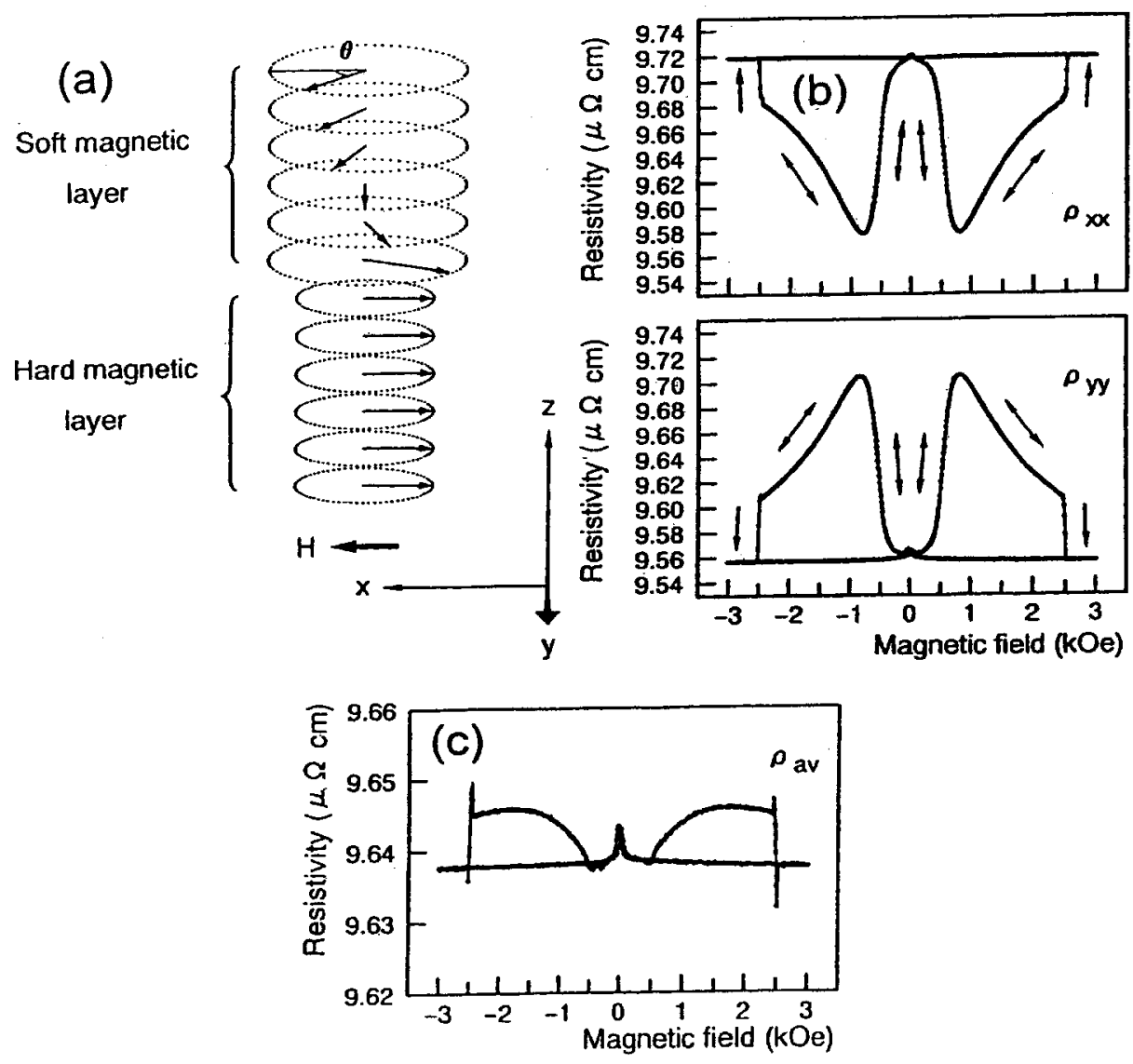

Fig. 2. (a) Illustration of a spring-magnet bilayer system. (b) Magnetoresistance curves of $\mathrm{NiFe}(30 \mathrm{~nm}) / \mathrm{CoSm}(100 \mathrm{~nm})$ with the electric current parallel and perpendicular to the magnetic field. (c) Average of two magnetoresistance curves in (b).

direction of magnetization and eventually two domain walls per each pad are produced artificially (Fig. $3 \mathrm{~b}$ ). The magnetoresistance of this system is negative and interpreted as the AMR effect. From the samples with different number of pads, the resistivity as a function of domain wall number is estimated as shown in Fig. 3c. The linear relation indicates that the domain walls are artificially well controlled. The negative magnetoresistance means that the contribution of the AMR effect is dominant and other contributions, such as from non-collinear spin structures, are too small to argue.

Very recently, extremely large MR effect has been reported for point contact systems, consisting of $\mathrm{Ni}$ wires [3] and $\mathrm{Fe}_{3} \mathrm{O}_{4}$ crystallites [4]. The very large MR ratios in moderate fields at room temperature are attractive also from technical points of view. It is theoretically supported that a domain wall confined in a very 
(a)
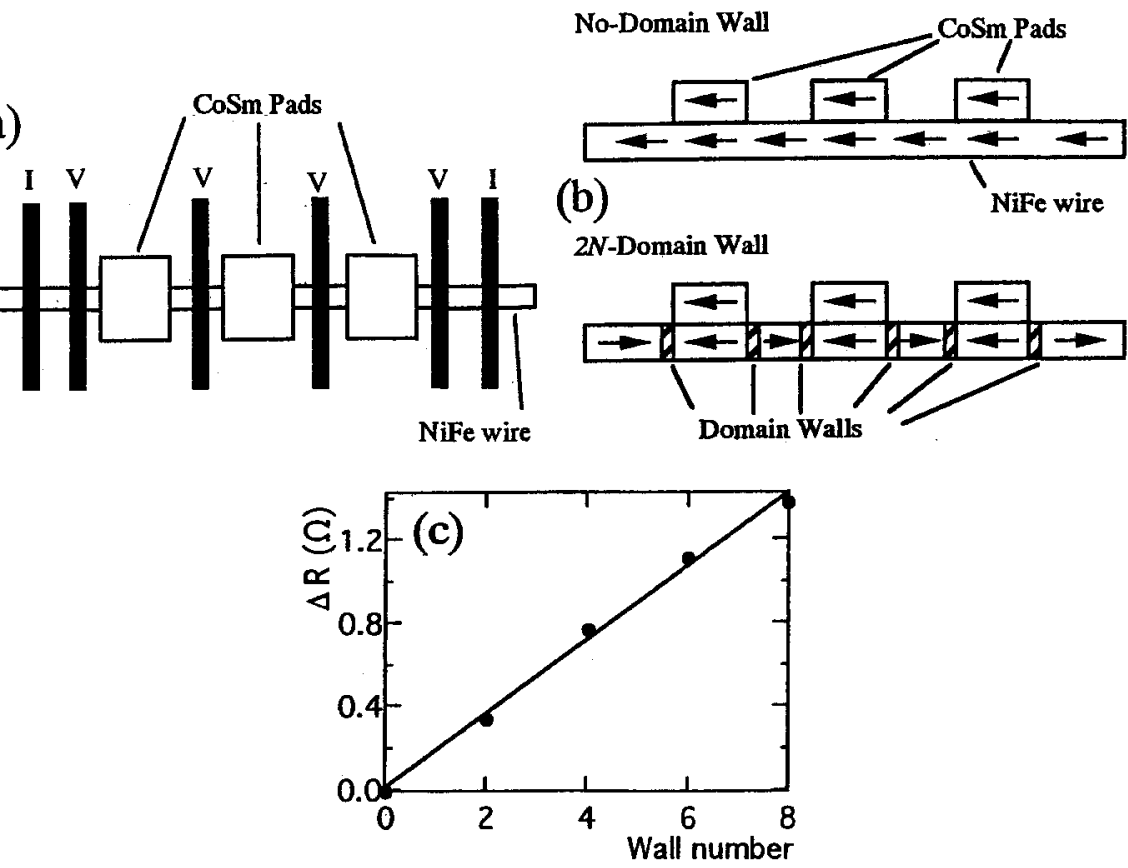

Fig. 3. (a) Schematic structure of NiFe wire with CoSm pads. (b) Illustration of non-wall state and $2 \mathrm{~N}$-wall state. (c) The negative resistance as a function of domain wall number.

narrow thickness, where the neighboring spins have large angles, can be a source for a great resistance, and subsequently may cause a very large MR effect [11]. Therefore the MR measurements on domain walls confined at point contact regions are of great interest. The present experiments introduced here however show that the contribution of domain walls to the resistivity is fairly small unless the size of the domain wall is greatly reduced. In order to explore novel MR systems, the preparation technique for nanoscale wire systems having point contacts are to be investigated.

\section{Vortex magnetization in NiFe dots}

It is common that ferromagnetic materials form multi-domain structure to reduce the magnetostatic energy. It is also well known that in very small particles of ferromagnetic materials, single domain structures are realized since the domain wall formation is energetically not favorable. On the other hand, in a circular dot of an appropriate size, a curling magnetic structure (vortex) can exist. In the vicinity of the dot center in a curling magnetic structure, the angle between adjacent spins becomes larger if the spin direction is confined in-plane. Therefore at the center 
of vortex structure, the magnetization will turn out-of-plane. Such a spot with perpendicular magnetization has been observed in circular NiFe dots by magnetic force microscopy (MFM) [12]. Figure 4 shows an example of MFM images for circular dots of permalloy with $1 \mu \mathrm{m}$ diameter. The dots show no other contrast than the central spots, which is an evidence of vortex magnetization structure with perpendicularly magnetized spots at each dot. The two types of contrast, white and black, correspond to up and down magnetization directions.
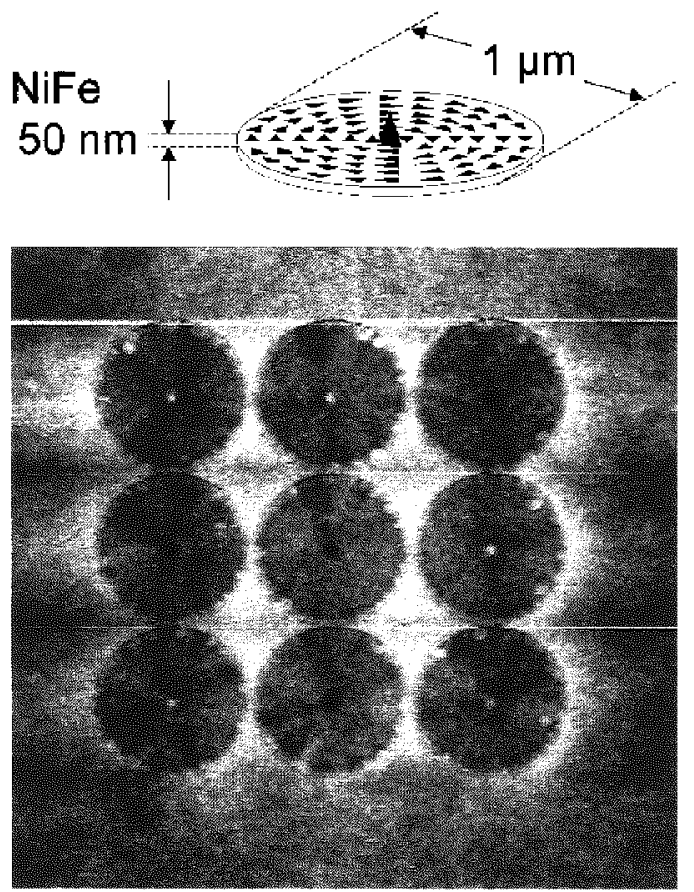

Fig. 4. Magnetic force microscopy image of NiFe circular dots with the diameter of $1 \mu \mathrm{m}$.

The reversal of perpendicular magnetization spots in a magnetization vortex is an interesting issue. The switching field is estimated by MFM. Since MFM observation is unable in the presence of a large magnetic field, the switching of the magnetization direction is observed after removing the external field. The sample used for this experiment is an array of 25 circular dots of $\mathrm{NiFe}$ with the diameter of $400 \mathrm{~nm}$ and the thickness of $50 \mathrm{~nm}$. By applying a strong external field, all magnetization spots are oriented in the field direction and accordingly the MFM image shows that all dots have the same contrast (black) at each center. MFM images were taken by varying the external field step by step. The number of dots with reversed magnetization spots is shown in Fig. 5a, as a function of applied field. From this curve, the average reversal field is determined to be about 4,000 Oe. 

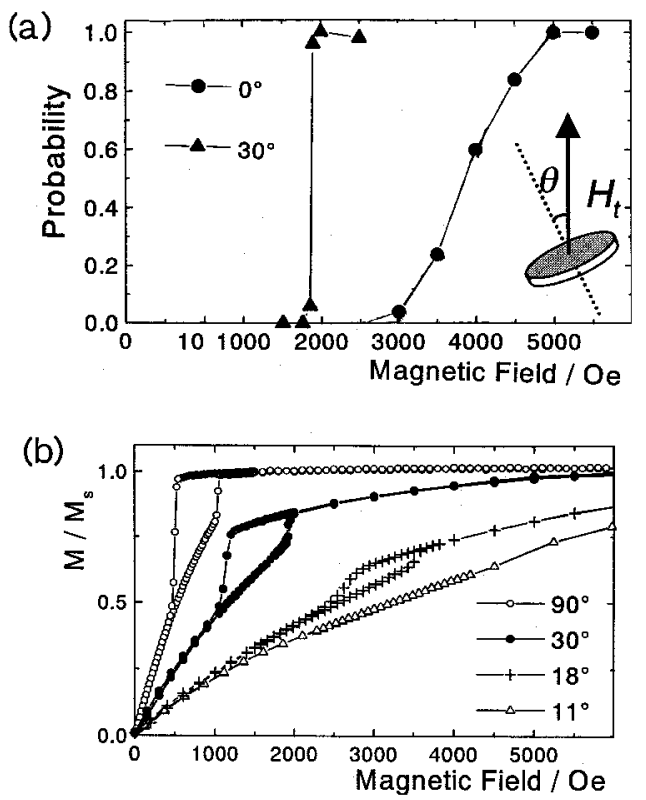

Fig. 5. (a) Number of switched dots among $25 \mathrm{NiFe}$ dots with $400 \mathrm{~nm}$ diameter by the application of external fields. The direction of external field is along the normal to the plane $\left(\theta=0^{\circ}\right)$ and $30^{\circ}$ from the normal. (b) Magnetization curves for permalloy dot arrays with $400 \mathrm{~nm}$ diameter. $\theta$ denotes the direction relative to the normal to the plane.

Magnetization curve of the dot arrays is measured by SQUID magnetometer. Figure $5 \mathrm{~b}$ shows that the saturation field for perpendicular field is about 10,000 Oe. The magnetization is gradually increasing from 0 to the saturation field, and no anomaly due to the reversal of perpendicular magnetization spot is observed around 4,000 Oe. It is reasonable, even if the reversal transition occurs abruptly, since the relative volume of the core spot is regarded to be very small.

If the external field is applied to an in-plane direction, the feature of the reversal phenomena is different. MFM measurements show that the reversal of the magnetization spots occurs sharply at about 2,000 Oe. This result is interpreted as the displacement of vortex core caused by the external field. Under an in-plane field, the vortex core moves from the center to the rim of the dot and finally extincts. If an external field, $H_{\mathrm{ex}}$ is applied at an angle of $30^{\circ}$ to the normal, the field component in the plane is $H_{\mathrm{ex}} \sin \theta$. Namely, the in-plane field for the annihilation of vortex core is 1,000 Oe. The magnetization curve for in-plane field exhibits a definite hysteresis, having a jump just before the saturation, which is supposed to correspond to the annihilation of vortex core. By comparing the saturation field and the switching field obtained from MFM measurement in Fig. 5, this hypothesis is verified. Thus the process of magnetization reversal for in-plane 
field is well understood: the vortex core has moved out from the dot. On the other hand, for perpendicular magnetic fields, the behavior of magnetization vortex core is not clarified yet. Further experiments and theoretical simulations are required.

\section{Summary}

Experimental studies on the following subjects are briefly surveyed and the usefulness of microfabrication techniques for magnetic materials is introduced.

1) Behaviors of a domain wall in a single wire system prepared by microfabrication technique were studied from resistivity measurements. The velocity of domain wall propagation is able to be estimated.

2) The contribution of domain wall to the conductivity were studied from measurements on an exchange spring bilayer system.

3) The existence of vortex magnetization with a perpendicularly magnetized spot was confirmed by MFM imaging for circular NiFe dots. Reversal process of the core magnetization for perpendicular and in-plane fields is argued.

\section{Acknowledgments}

The author would like to thank K. Mibu, T. Ono, T. Nagahama, K. Shigeto, T. Okuno, K. Miyake, Y. Suzuki, and R. Hassdorf for their collaboration. This work has been supported by the Grants-in-aid for scientific research from Monbusho and a NEDO project.

\section{References}

[1] E.M. Chudnovsky, J. Tejada, Macroscopic Quantum Tunneling of the Magnetic Moment, Cambridge University Press, Cambridge 1998; G. Tatara, H. Fukuyama, Phys. Rev. Lett. 78, 3773 (1997).

[2] B. Barbara, L. Thomas, F. Lionti, I. Chiorescu, A. Sulpice, J. Magn. Magn. Mater. 200, 167 (1999).

[3] N. Garcia, H. Rohrer, I.G. Saveliev, Y.W. Zhao, Phys. Rev. Lett. 85, 3053 (2000).

[4] J.J. Versluijs, M.A. Bari, J.M.D. Coey, Phys. Rev. Lett. 87, 026601 (2001).

[5] T. Ono, H. Miyajima, K. Shigeto, T. Shinjo, Appl. Phys. Lett. 72, 1116 (1998).

[6] T. Ono, H. Miyajima, K. Shigeto, K. Mibu, N. Hosoito, T. Shinjo, Science 284, 468 (1999).

[7] K. Shigeto, T. Okuno, T. Shinjo, Y. Suzuki, T. Ono, J. Appl. Phys. 88, 6636 (2000).

[8] J.P. Gregg, W. Allen, K. Ounadjela, M. Viret, M. Hehn, S.M. Thompson, J.M.D. Coey, Phys. Rev. Lett. 77, 1580 (1996); K. Hong, N. Giordano, J. Phys. Condens. Matter. 8, L301 (1996); Y. Otani, K. Fukamichi, O. Kitakami, Y. Shimada, B. Pannetier, J.P. Nozieres, T. Matsuda, A. Tonomura, Mater. Res. Soc. Symp. Proc. 475, 215 (1997). 
[9] K. Mibu, T. Nagahama, T. Shinjo, T. Ono, Phys. Rev. B 58, 6442 (1998).

[10] T. Nagahama, K. Mibu, T. Shinjo, J. Appl. Phys. 87, 5648 (2000).

[11] J. Mathon, M. Vileret, A. Umerski, to be published in J. Magn. Magn. Mater.

[12] T. Shinjo, T. Okuno, R. Hassdorf, K. Shigeto, T. Ono, Science 289, 930 (2000).

[13] T. Okuno, K. Shigeto, T. Ono, K. Mibu, T. Shinjo, to be published in J. Magn. Magn. Mater. 